\title{
Sleep deprivation does not affect neuronal susceptibility to mild traumatic brain injury in the rat
}

\author{
Aimee M Caron \\ Richard Stephenson \\ Department of Cell and Systems \\ Biology, University of Toronto, \\ Toronto, ON, Canada
}

This article was published in the following Dove Press journal:

Nature and Science of Sleep

22 June 2015

Number of times this article has been viewed

\begin{abstract}
Mild and moderate traumatic brain injuries (TBIs) (and concussion) occur frequently as a result of falls, automobile accidents, and sporting activities, and are a major cause of acute and chronic disability. Fatigue and excessive sleepiness are associated with increased risk of accidents, but it is unknown whether prior sleep debt also affects the pathophysiological outcome of concussive injury. Using the "dark neuron" (DN) as a marker of reversible neuronal damage, we tested the hypothesis that acute (48 hours) total sleep deprivation (TSD) and chronic sleep restriction (CSR; 10 days, 6-hour sleep/day) affect DN formation following mild TBI in the rat. TSD and CSR were administered using a walking wheel apparatus. Mild TBI was administered under anesthesia using a weight-drop impact model, and the acute neuronal response was observed without recovery. DNs were detected using standard bright-field microscopy with toluidine blue stain following appropriate tissue fixation. DN density was low under home cage and sleep deprivation control conditions (respective median DN densities, $0.14 \%$ and $0.22 \%$ of neurons), and this was unaffected by TSD alone ( $0.1 \%)$. Mild TBI caused significantly higher DN densities $(0.76 \%)$, and this was unchanged by preexisting acute or chronic sleep debt (TSD, $0.23 \%$; CSR, $0.7 \%$ ). Thus, although sleep debt may be predicted to increase the incidence of concussive injury, the present data suggest that sleep debt does not exacerbate the resulting neuronal damage.
\end{abstract}

Keywords: sleep deprivation, concussion, traumatic brain injury, dark neuron, neurodegeneration, rat cortex

\section{Introduction}

Insufficient or poor-quality sleep is associated with pronounced impairments in mammalian neurobehavioral function. ${ }^{1}$ In recent years, a substantial body of evidence has accumulated in support of a role for sleep in the maintenance of synaptic structure and function, ${ }^{2}$ but less attention has been given to the question of whether neuronal cellular integrity is dependent on sleep and impaired by sleep deprivation. ${ }^{3-8}$ Two important questions arise in the latter context: i) Does sleep deprivation affect neuronal integrity or viability? ii) Does sleep deprivation influence the susceptibility of neurons to traumatic insult? Current data pertaining to both of these questions are inconclusive and inconsistent.

There is limited and contradictory evidence relating to the hypothesis that sleep deprivation can induce neuronal cell death. Markers of DNA damage and neuronal degeneration were unchanged after 5-14 days of total sleep deprivation (TSD) ${ }^{4}$ or 4 days of rapid eye movement sleep deprivation (REMSD). ${ }^{6}$ However, REMSD for 6 days and 10 days resulted in a temporary increase in markers of apoptosis and neuronal
Correspondence: Richard Stephenson Department of Cell and Systems Biology, University of Toronto, Ramsay Wright Laboratories, 25 Harbord Street, Toronto, ON, Canada M5S 3G5

Tel + I 416978349 I

Email richard.stephenson@utoronto.ca 
damage in some brainstem nuclei of rats. ${ }^{3}$ Thus, REMSD may promote localized neurodegeneration but only after prolonged deprivation, and only in brainstem nuclei that are likely to be metabolically stressed by prolonged wakefulness. It is noteworthy that neurodegeneration was first observed long after the systemic physiological deficits associated with sleep loss are apparent, and it is therefore unlikely that the physiological and neurobehavioral consequences of sleep debt $^{9}$ are a direct result of cell death in associated neural control circuits. The rapid and apparently complete recovery of rats following prolonged TSD (even in animals exhibiting profound physiological dysfunction before the end of the procedure $)^{10}$ further argues against an underlying mechanism that involves permanent brain cell damage.

The above studies do not rule out the possibility that TSD may cause reversible, nonlethal changes in neuronal structure and function. The "dark neuron" (DN) is a histological marker of reversible cell damage; in the absence of ischemia or excitotoxicity, DNs are capable of recovery and begin to regain their normal staining properties in as little as 1 hour post-insult. ${ }^{11-14}$ There are reports in the literature that support the hypothesis that sleep deprivation can cause this kind of neuronal damage. Crile ${ }^{15}$ described diffuse hyperchromatism (increased Nissl stain uptake) in cerebellar Purkinje cells following 4-5 days of TSD in rabbits and apparent reversal of this effect following recovery sleep. Eiland et $\mathrm{al}^{5}$ reported argyrophilia (amino-cupric silver staining) in the supraoptic nucleus (SON) of rats after 2 days of TSD but equivocal results in other brain regions. Biswas et $\mathrm{al}^{3}$ reported an increase in silver staining in some brainstem nuclei following 6 or more days of REMSD in rats.

The morphological features of DN that are visible under a light microscope include compaction (cell shrinkage), hyperbasophilia (high uptake of basic histological stains), argyrophilia (uptake of silver stains), corkscrew-like dendrites, and a triangular-shaped soma. ${ }^{11,16-21} \mathrm{DN}$ can form in response to a variety of physical and chemical stimuli, including (but not limited to) mechanical trauma $a^{12,14,22,23}$ and ischemia. ${ }^{7,24}$ The latter stimuli are important not only because of their relevance to pathophysiological conditions such as concussion and $\mathrm{TBI}^{25}$ but also because they can promote the formation of artifactual DN during tissue processing. Both mechanical trauma and global ischemia are present during autopsy and can cause DN formation in unfixed or partially fixed tissue. Cammermeyer ${ }^{17}$ developed a method of perfusion fixation with aldehyde fixatives that reliably produces tissue samples free of DN. This method requires that brains be perfused immediately postmortem and that autopsy be delayed for more than 24 hours. He noted that "after the lapse of $24 \mathrm{~h}$ the neurons are not yet quite fixed and many of them will react to trauma" (p. 249). ${ }^{17}$ Formaldehyde (mainly in the form of methylene glycol) rapidly penetrates tissues and impedes autolysis, but fixation of the tissue through the formation of methylene cross-linkages is slow and may take several days at low temperature. ${ }^{26,27}$ Unfortunately, none of the previous studies of DN formation in sleep-deprived animals ${ }^{3,5,15}$ used an adequate fixation protocol, rendering their results and conclusions open to question.

There are an estimated ten million traumatic brain injuries (TBIs) per year (approximately one in every 3 seconds) worldwide. ${ }^{28}$ The causes are primarily linked to falls, automobile accidents, and sporting activities, and the severity and time course of symptoms vary widely. ${ }^{29}$ Causes and consequences of TBI are potentially linked to sleep in four ways: i) Sleep debt may be a risk factor for TBI. Drowsiness and excessive daytime sleepiness are risk factors for automobile and occupational accidents, ${ }^{30,31}$ suggesting that the growing prevalence of sleep debt might be accompanied by an increased incidence of TBI, although this has not yet been confirmed. ii) Conversely, TBI may be a risk factor for sleep dysregulation, since disruption of sleep-wake pattern is a recognized symptom of TBI and post-concussion syndrome. Sleep disturbances are reported in approximately half of young people following mild TBI and concussion. ${ }^{32}$ iii) Sleep may be implicated in the recovery process following concussive or cerebrovascular brain injury, ${ }^{33-35}$ although remediation of sleep disturbance was not associated with improved functional outcomes. ${ }^{36}$ iv) Preexisting sleep debt may influence the severity of initial damage resulting from a mild traumatic injury. The latter hypothesis has not been subjected to experimental test and is a focus of the present study.

The primary aims of the present study were to determine, with appropriate controls and tissue fixation technique, whether TSD causes DN formation in rat sensorimotor cortex, and whether sleep debt (caused by acute TSD or chronic sleep restriction [CSR]) affects the vulnerability of neurons to mild TBI.

\section{Materials and methods}

\section{Animals and experimental protocol}

All procedures conformed to guidelines of the Canadian Council on Animal Care and were approved by the animal care committee of the University of Toronto. Studies were performed using 68 male Sprague Dawley rats divided into seven groups: perfusion-fixed home cage control 
(HCC; $n=12$, body weight range 369-434 g), perfusion-fixed stimulus control (SDC; $\mathrm{n}=6,394-435 \mathrm{~g}$ ), immersion-fixed positive control (IMM; $n=13,351-717 \mathrm{~g}$ ), 48 hours of TSD $(\mathrm{n}=8,283-390 \mathrm{~g})$, TBI $(\mathrm{n}=6,339-399 \mathrm{~g}), 48$ hours of TSD followed by TBI (TSD-TBI; $n=13,314-438 \mathrm{~g}$ ), and 10 days of CSR (daily 18-hour sleep deprivation and 6-hour sleep opportunity) followed by TBI (CSR-TBI; $\mathrm{n}=10,373-439 \mathrm{~g}$ ).

In order to avoid inadvertent neuronal damage due to depression of the cortical surface by electrode screws, the animals were not instrumented with electroencephalographic (EEG) electrodes. Other details of the experimental protocol (laboratory setting, animal maintenance, sleep deprivation technique) prior to brain tissue autopsy and processing were as described in detail previously. ${ }^{37}$ All animals were acclimatized to the laboratory setting, and a 12:12 light/dark cycle for a minimum of 1 week before the start of experiments. Animals were provided with unrestricted access to food (standard chow) and water throughout the study.

Briefly, sleep deprivation was imposed using a walking wheel revolving at $6.4 \mathrm{~cm} \cdot \mathrm{s}^{-1}$. The wheel was used in an intermittent rotation mode ( 8 seconds on and 8 seconds off cycle). In TSD, wheel rotations began at lights on (zeitgeber time, ZT0) and ended 48 hours later at the end of the dark period. In CSR, daily wheel rotations began at ZT6 (midday) and ended at the end of the dark period (ZT24). Thus, the wheel was immobile for 6 hours (ZT0-ZT6), providing a daily sleep opportunity during the early light phase. This was repeated on each of 10 consecutive days.

Mild TBI was produced using the impact injury model described and characterized by Foda and Marmarou ${ }^{38}$ and Marmarou et al. ${ }^{39}$ This method causes diffuse brain injury without focal lesions. Briefly, the anesthetized rat (isoflurane; $1 \%-2 \%$ in air $/ \mathrm{O}_{2}$ mix) was placed prone with the dorsal surface of the cranium horizontal. The scalp was retracted, and an aluminum disk (10 $\mathrm{mm}$ diameter, $3 \mathrm{~mm}$ thickness) was affixed using dental acrylic cement to the surface of the cranium, centered on the sagittal suture, midway between the lambda and bregma intersections. This disk served to prevent bone fracture and subdural hematoma. A Plexiglas tube (20 mm internal diameter) was positioned vertically above the cranial disk and used as a guide for a brass projectile (impact surface $6 \mathrm{~mm}$ diameter). In preliminary studies, we determined that a weight of $500 \mathrm{~g}$ dropped from a height of $100 \mathrm{~cm}$ was sufficient to produce significant neuronal trauma (as indicated by DN formation) without macroscopically detectable contusive injury, and this procedure was used in all rats subjected to TBI in the present study.

\section{Brain tissue autopsy and processing}

All animals were processed in the interval ZT0-ZT6 (immediately following the end of the final sleep deprivation stimulus in TSD and CSR groups). Prior to sacrifice, animals received either an intraperitoneal dose of sodium pentobarbital $(100 \mathrm{mg} / \mathrm{kg})$ or inhalable isoflurane $(1 \%-2 \%$ in air/ $\mathrm{O}_{2} \mathrm{mix}$ ) anesthetic, followed by an intracardiac injection of $0.1 \mathrm{~mL}$ of heparinized (323 units $/ \mathrm{mL}$ ) saline. Since the objective of control studies was to avoid artifactual DN formation (ie, to yield brain slices with no $\mathrm{DN}$ ), a positive control procedure was applied in every animal to confirm that histological procedures were adequate for the demonstration of DN when present. Thus, in every animal prior to sacrifice, a $0.4 \mathrm{~mm}$ hole was drilled in the right frontal cranium (coordinates, expressed relative to bregma, antero-posterior $-2 \mathrm{~mm}$, medio-lateral $2 \mathrm{~mm}$ ) for administration of a pin prick (size 000 insect pin, diameter $0.25 \mathrm{~mm}$ to a depth of $4 \mathrm{~mm}$ ) which is known to reliably cause formation of many DNs in tissue adjacent to the pin track (Figure 1). Rats were then overdosed with additional anesthetic until cardiac activity ceased (analyses showed that the type of anesthetic had no statistical effect on DN counts). Brains of IMM animals were immediately carefully autopsied and submerged in a

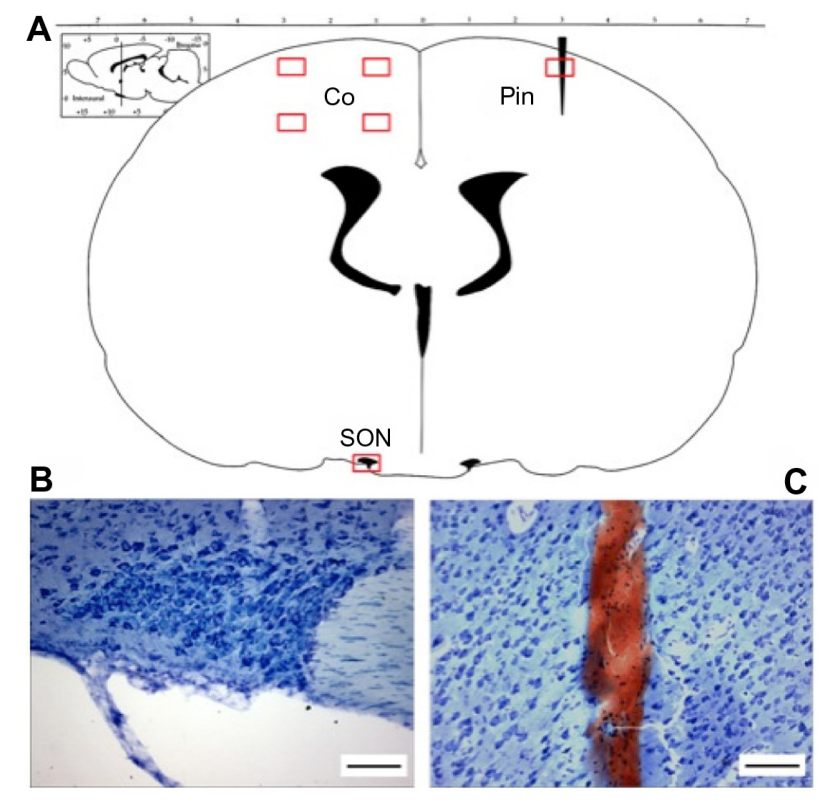

Figure I ROls used in quantitative analysis of DNs in coronal sections of the rat brain. Notes: (A) Schematic of a coronal section through the brain at AP $-2 \mathrm{~mm}$ relative to bregma (adapted from Ref 57). $\square$ indicate ROls $(600 \mu \mathrm{m} \times 400 \mu \mathrm{m})$ used for cell counts in the Co, SON, and in the Pin. (B) SON ( $\times 100$ magnification), illustrating generalized hyperbasophilia and the absence of obvious compacted DN. (C) Photomicrograph of cortical neurons in a pin-prick positive control at $\times 100$ magnification. DNs (basophilic with a compacted triangular shape) are visible in the tissue adjacent to the pin lesion. Tissues stained with toluidine blue. Scale bars, $100 \mu \mathrm{m}$.

Abbreviations: ROI, region of interest; DN, dark neuron; Co, cortex; SON, supraoptic nucleus; Pin, cortex pin-prick perilesion. 
solution of $4 \%$ formaldehyde in phosphate-buffered saline (PFA; pH 7.4) at room temperature, and chilled to $4^{\circ} \mathrm{C}$ for at least 48 hours. All other rats were perfused transcardially with at least $100 \mathrm{~mL}$ of $0.9 \%$ saline, followed by $300 \mathrm{~mL}$ of $4 \%$ PFA for a maximum of 10 minutes. Time between cardiac arrest and start of PFA perfusion was approximately 5 minutes. Perfusates were at room temperature and were allowed to flow without restriction by gravity from $100 \mathrm{~cm}$ above the heart. Animals were left untouched in the supine position following PFA perfusion and maintained at $4{ }^{\circ} \mathrm{C}$ for 3 days post-perfusion. Brains were then carefully autopsied and postfixed in $4 \%$ PFA at $4^{\circ} \mathrm{C}$ for at least an additional 24 hours.

After fixation, tissues were cryoprotected with $30 \%$ sucrose in phosphate-buffered saline at $4{ }^{\circ} \mathrm{C}$ for at least 48 hours, with a fresh solution change after approximately 24 hours. The brains were then flash frozen by immersion in 2-methylbutane (isopentane) kept on dry ice, and then stabilized using Tissue-Tek CRYO-O.C.T. compound (Fisher Scientific, Ottawa, Ontario, Canada). Coronal sections (bregma $-0.8 \mathrm{~mm}$ to bregma $-1.5 \mathrm{~mm}$ ) were sliced at $40 \mu \mathrm{m}$ (Feather blades, Fisher Scientific 12-634-1C) and mounted using 30\% sucrose solution onto $3 \%$ gelatincoated glass slides, which were allowed to dry naturally in the dark before staining. A single slice containing the SON was used for analysis of the sensorimotor cortex and SON. A separate slice containing the pin-prick track was used as a positive control for DN.

In a pilot study, three stains were evaluated: toluidine blue (TB), Nissl (cresyl violet), and silver (FD Neurosilver kit). Silver stain was found to be inconsistent; background staining was variable, and DNs were sometimes difficult to identify, even in damaged tissue (pin prick and IMM fixed). Silver staining is known to be prone to poor selectivity and reproducibility, ${ }^{40}$ problems that were not alleviated by the use of a standardized kit, and silver-stained slides were therefore excluded from further analysis. Nissl and TB were found to reliably stain both normal cells and DNs. However, TB yielded lower variability of background staining and better resolution of $\mathrm{DN}$, and the following analysis is therefore based only on TB-stained tissues.

A working solution of TB was prepared using $0.5 \mathrm{~g}$ of tolonium chloride (toluidine blue O; Sigma-Aldrich Canada Co, Oakville, ON, Canada) and $500 \mathrm{~mL}$ of $1 \%$ acetic acid solution. Dry slides were submerged in this $0.1 \%$ solution of TB for 40 seconds at room temperature before being well rinsed with distilled water and allowed 24 hours to dry at room temperature in the dark. Slides were then dehydrated for
2 minutes in $100 \%$ ethanol, cleared with xylene for 5 minutes, covered with Permount (Fisher Scientific Co, Ottawa, ON, Canada), and coverslipped.

\section{Visualization of tissue preparations}

Stained slides were visualized under a light microscope (BX50W1 Olympus Microscope, Carsen Group Inc., Markham, ON, Canada), and pictomicrographs were obtained using a high-resolution digital camera (3.3 RTV Micro Publisher, QIMAGING, Burnaby, BC, Canada).

Areas of the coronal slice that were selected for examination (regions of interest, ROIs) included four areas of the left sensorimotor cortex (Figure 1), one ROI of the ipsilateral hypothalamus containing the SON, and one ROI of the contralateral cortex containing a pin prick as a positive control for DN. The four ROIs in the sensorimotor cortex each comprised an area of $400 \mu \mathrm{m} \times 600 \mu \mathrm{m}$, and were delineated using a standardized procedure for alignment of the field of view in every brain slice at $\times 40$ magnification. Data from the four cortical areas were pooled for quantitative analysis. Cell counts were made at $\times 100$ magnification (Figure 2 ).
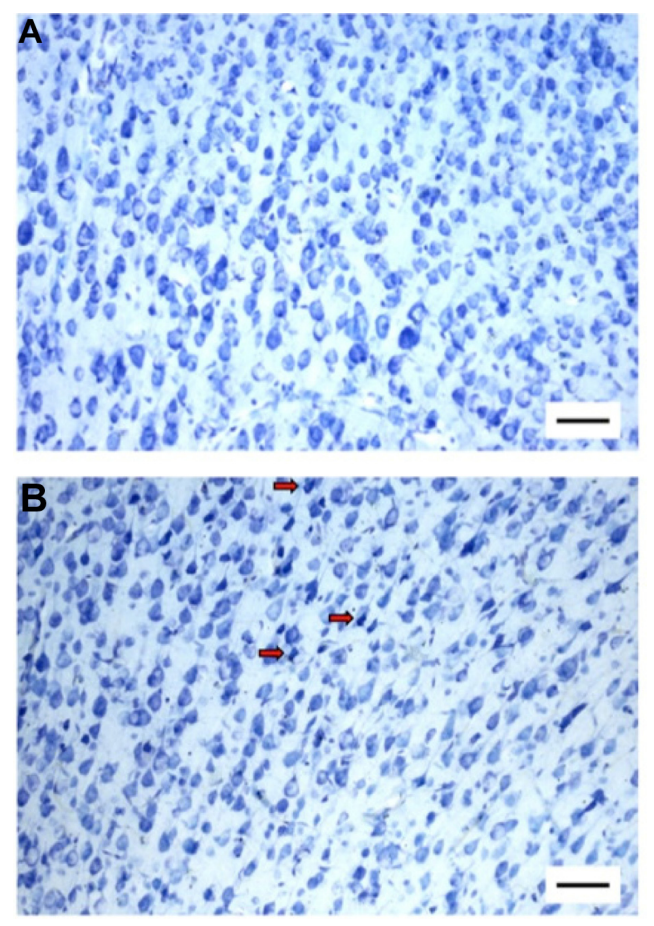

Figure 2 Artifactual DN formation in the cortex following immersion fixation. Notes: (A) Photomicrograph $(\times 100)$ of a representative perfusion-fixed cortical region of interest (HCC group) demonstrating the absence of DN. (B) Photomicrograph $(\times 100)$ of a representative immersion-fixed cortical region of interest (IMM group), showing numerous DNs ( $\Rightarrow$ heads) scattered at random among normal neurons. Stained with toluidine blue. Scale bars, $50 \mu \mathrm{m}$.

Abbreviations: DN, dark neuron; HCC, perfusion-fixed home cage control; IMM, immersion-fixed positive control. 
The visual identification of DN is to some extent subjective, and to minimize inconsistency, all tissue examination and cell quantification were performed by a single experimenter (AMC) using a randomized blind protocol, and using three scoring criteria: color, shape, and size. The inclusion of shape and size criteria precluded a simple automated analysis involving background subtraction and gray-scale threshold detection. Although there will undoubtedly be intra-scorer and inter-scorer variability using this approach, we assume that such variability is randomly distributed across experimental groups.

1. Normal neurons: appear relatively light in color with a round or teardrop shape and a size typical of the majority of neurons in the ROI.

2. DNs: appear dark in color with an angular shape and a compacted size in comparison to the normal neurons in the ROI.

For each ROI, the number of neurons of each type was counted using the ImageJ manual cell counter plugin (Javabased open-source software, http://rsbweb.nih.gov/ij/). Neurons touching the right edge or bottom edge of a counting grid were not included in the final cell counts, while those touching the top and left sides of the grid were included. ${ }^{41}$

\section{Particle analysis}

The three criteria used in visual scoring of normal $(\mathrm{n}=723)$ and $\mathrm{DN}(\mathrm{n}=126)$ cell types were evaluated by quantitative particle analysis (ImageJ, Java-based open-source software, http:// rsbweb.nih.gov/ij/) in a sample of visually scored cells taken from positive control samples (IMM and pin-prick ROI).

\section{Color}

The color of an individual neuron was sampled from an area of the soma that did not contain the nucleus. $R G B$ pixels were converted to brightness $(\mathrm{Br})$ values using the formula $\mathrm{Br}=(\mathrm{R}+\mathrm{G}+\mathrm{B}) / 3$, with lower values representing a darker color (value of 0 represents extreme black and 255 represents extreme white). To account for differences in staining between slides, brightness values were expressed as a percentage of normal cell brightness (averaged from a sample of 50 normal neurons on every slide).

\section{Shape and area}

Automatic edge detection was applied to 8-bit monochrome images and used to outline neurons. Cell shape ("soma form factor", FF) was quantified as the normalized ratio of area to perimeter ( $\mathrm{FF}=4 \pi A / p^{2}$, where $A$ is area $\left[\mu \mathrm{m}^{2}\right]$ and $p$ is perimeter $[\mu \mathrm{m}])$, so that in a true circle, this ratio is one. ${ }^{42}$
Values closer to zero indicate increasingly elongated shapes (eg, $\mathrm{FF}$ of an equilateral triangle is 0.777 ).

\section{Statistical analysis}

Statistical analyses were performed using Sigmastat Statistical Software (version 3.5, Systat Software Inc., Point Richmond, CA, USA) or GraphPad Prism (version 6.0f, GraphPad Software Inc., La Jolla, CA, USA). When appropriate, normally distributed homoscedastic paired data were tested using the paired $t$-test. In cases in which data were not normally distributed, a nonparametric Kruskal-Wallis test, with post hoc Dunn's multiple comparisons test, was used to compare three or more groups. For two-group comparisons, the Wilcoxon signed rank test was used for matched pairs, and Mann-Whitney rank sum test was used for independent samples. Values are expressed as means \pm standard error of the mean or median \pm interquartile range (IQR). Effect size was quantified using the Hodges-Lehmann estimator with 95\% confidence intervals (CIs). The fiducial level of statistical significance was set at $P \leq 0.05$.

\section{Results \\ Quantification of morphological characteristics used as DN scoring criteria}

Samples of DN from both immersion-fixed and pin-prick positive control slides were significantly less bright than normal neurons in the same slides (relative brightness $65.9 \% \pm 1.27 \%$; paired $t$-test, $P<0.001$ ). DNs were significantly less circular (more elongated) than normal neurons (FF, median [IQR], 0.58 [0.55-0.61] and 0.68 [0.63-0.73], in DNs and normal neurons, respectively; Wilcoxon signed rank test, $P<0.001$ ). The area of DN was significantly smaller than normal neurons (98.0 [92.4-106.5] and 159.5 [146.1-184.1] $\mu \mathrm{m}^{2}$, respectively; Wilcoxon signed rank test, $P=0.001$ ).

\section{Positive and negative controls}

The cortex of perfusion-fixed brains autopsied from HCC animals had very few DNs (and usually none; median [IQR], 0.14 [0.07-0.27]\% of neurons in ROI). Likewise, SDC rats exhibited very low DN counts $(0.22[0.10-0.40] \%)$. These controls were not statistically significantly different (MannWhitney test, $P=0.45$ ). DNs were observed surrounding the cortical pin-prick lesion in every case (Figure 1), thereby validating the tissue preparation and histological techniques and confirming that DN-free samples were legitimate. In contrast, despite taking great care during immersion fixation procedures, the brains of the IMM group contained appreciable 
numbers of DNs $(1.41[0.78-2.21] \%)$ that were randomly dispersed among normal neurons (Figure 2), confirming that incompletely fixed tissue is susceptible to artifactual DN formation during autopsy. . $^{17,20}$

There was no difference in the number of DNs in HCC animals anesthetized with pentobarbital or isoflurane (unpaired $t$-test, $P=0.283$ ).

\section{Supraoptic nucleus}

Compacted DN could not be identified in the SON of any animal, including animals whose brains were immersionfixed (in which DNs were readily visible in the cortex). To increase the probability of forming DNs in the SON, six of the IMM animals were subjected to intentional trauma in the approximate region of the SON, while the brain was intact and unfixed. A brass weight of either $1 \mathrm{~g}$ or $50 \mathrm{~g}$ was placed on the ventral surface of the brain for 2 minutes prior to immersion fixation in 4\% PFA. Despite this mechanical insult, DNs could not be distinguished from normal neurons in the SON, even when DNs were conspicuous in hypothalamic areas adjacent to the SON.

Neurons of the SON stained more darkly $(\mathrm{Br}=84.3 \pm 5.1)$ than normal cortical neurons $(\mathrm{Br}=111.0 \pm 7.7)$ (paired $t$-test, $P=0.007)$. Cell area and FF of SON neurons were equivalent in HCC and IMM ( $P=0.11$ and 0.89 for area and FF, respectively). Thus, all three of the criteria used in this study failed to discriminate between normal cells and DNs in the SON. SON data were therefore excluded from further analysis.

\section{Cortex: effect of TSD}

Forty-eight hours of TSD in the walking wheel apparatus produced no significant difference in cortex DN density $(0.10$ [0.08-0.32]\%) compared to either HCC or SDC animals

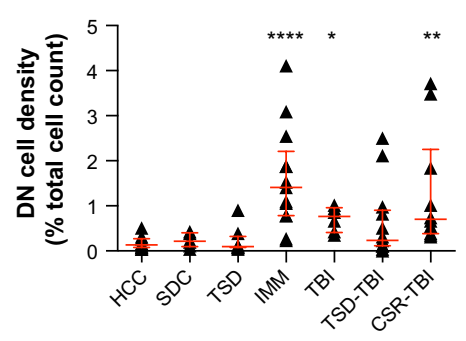

Figure 3 DN counts in rat sensorimotor cortex.

Notes: DN density is expressed as \% of total cell count in the combined cortex regions of interest. Symbols indicate individual animals, error bars (壬) denote median \pm interquartile range, and asterisks indicate a statistically significant difference vs HCC (Kruskal-Wallis test followed by Dunn's multiple comparisons test: $* P<0.05, * * P<0.01$, and $* * * * P<0.000$ I).

Abbreviations: DN, dark neuron; HCC, perfusion-fixed home cage control; SDC, perfusion-fixed stimulus control; TSD, total sleep deprivation; IMM, immersionfixed positive control; TBI, traumatic brain injury; TSD-TBI, TSD followed by TBI; CSR-TBI, chronic sleep restriction followed by TBI.
(Kruskal-Wallis test, $P=0.623$; Figure 3). Hodges-Lehmann estimator was 0 [ $95 \% \mathrm{CI},-0.1566$ to 0.07828$]$ for HCC vs TSD and $-0.078[-0.3131$ to 0.2740$]$ for SDC vs TSD.

\section{Cortex: effect of TBI, with and without prior sleep debt}

Induction of mild TBI by the weight-drop protocol successfully caused increased formation of DN in the cortex; DN density was significantly greater in the TBI group $(0.76$ [0.41-0.96]\%) than in HCC and SDC groups (Kruskal-Wallis test, $P<0.0001)$, confirmed by Hodges-Lehmann estimators (0.587 [0.274-0.822] and 0.489 [0.117-0.822] for TBI vs HCC and TBI vs SDC, respectively). Prior sleep debt induced by 48 hours of TSD or 10 days of CSR had no statistically significant effect on DN density vs TBI alone (TSD-TBI, 0.23 [0.12-0.90]\%; CSR-TBI, 0.70 [0.38-2.25]\%; Kruskal-Wallis test, $P=0.083$; Figure 3 ). The absence of effect was supported by Hodges-Lehmann estimators, which overlapped zero in both TSD-TBI vs TBI $(-0.313$ [ -0.44 to 0.391$])$ and CSRTBI vs TBI (0.059 [ -0.391 to 2.544$])$. However, a prominent feature of the responses to TBI was a higher inter-animal variability in DN density compared with controls (TBI vs HCC groups in Figure 3). Prior sleep debt appeared to increase this variability (TBI vs TSD-TBI and CSR-TBI groups in Figure 3), but this effect could not be evaluated statistically.

\section{Discussion}

The present study shows for the first time that preexisting sleep debt does not affect the vulnerability of cortical sensorimotor neurons to the immediate effects of mild TBI. We also refute the hypothesis that acute (48 hours) TSD causes $\mathrm{DN}$ formation in the sensorimotor cortex of the rat, corroborating conclusions reached in previous research., ${ }^{3,5}$

In a previously published study on the effect of TSD on DN formation, ${ }^{5}$ rat brains were processed or manipulated in less than 24 hours following perfusion of formaldehyde fixative, which is known to cause artifactual formation of DN. ${ }^{16-18}$ Some of the animals in that study ${ }^{5}$ were described as exhibiting "a diffuse, dust-like reactive staining ... in a wide area of dorsal cortex", but a statistically significant effect of TSD could not be demonstrated. ${ }^{5}$ The results of the present study are consistent with this negative statistical conclusion and reinforce the suggestion that the above data may have been compromised by inappropriate tissue-handling technique. Samples taken from the IMM group in this study show that this issue is not trivial, since DN counts due to careful autopsy of unfixed brain were at least as great as those caused by impact trauma in the TBI group (Figure 3). 
In contrast, by delaying autopsy for 3 days after perfusion fixation, control group brains (HCC and SDC) were shown to contain very few or no DN (Figures 2 and 3). The positive controls (pin prick) confirmed this to be a genuine observation of absence, and not faulty technique.

Biswas et $\mathrm{al}^{3}$ observed argyrophilia in brainstem nuclei under all conditions, including control, but nevertheless reported significantly greater DN in rats subjected to prolonged REMSD ( $\geq 6$ days, by the flowerpot technique) than in control animals. Notably, the enhancement of argyrophilia was absent following 3 days of subsequent sleep recovery. However, the inadequate fixation technique used in that study admits two possible conclusions: that REMSD was the direct cause of neuronal damage and that prolonged REMSD enhanced the susceptibility of brain to artifactual damage. The present study does not support the latter interpretation, since TBI did not cause higher DN counts in animals with sleep debt (compared with those subjected to TBI without prior TSD or CSR).

Eiland et $\mathrm{al}^{5}$ examined whole-brain argyrophilia following prolonged TSD (8-10 days using the disk-over-water technique). Argyrophilia was observed in all animals, but a statistically significant difference between TSD and control was found only in the SON. Unfortunately, our attempts to verify this result were unsuccessful because we found that DN could not be reliably identified in SON using the TB and Nissl stains. The magnocellular neurosecretory cells of the SON contain large quantities of rough endoplasmic reticulum, which is basophilic, and therefore, the SON neurons stain darkly even under control conditions (Figure 1). Eiland et $\mathrm{al}^{5}$ reported the same result using Nissl stain. We found no evidence of cell compaction (reduced size and circularity) in SON neurons, even in IMM samples or following application of pressure to the ventral surface of the unfixed brain. This failure of the positive control precludes a conclusion, and further research will be required to confirm or refute the report by Eiland et al. ${ }^{5}$

In a previous study, ${ }^{37}$ we found that EEG- and electromyogram (EMG)-instrumented rats subjected to 48 hours of TSD and 10 days of CSR accumulated sleep deficits of 21 hours and 55 hours, respectively, and we assume similar sleep deficits under identical conditions in the TSD and CSR groups of uninstrumented rats in the present study. This level of sleep debt, while not severe, is nevertheless sufficient to elicit appreciable neurobehavioral and physiological effects. In rats, TSD of 1-4 days is associated with a progressive suppression of behavioral and EEG sleep-wake homeostatic responses ${ }^{43}$ and a rise in body temperature and metabolic rate. ${ }^{9}$ Acute TSD also affects neurobehavioral performance, learning and memory, and changes in synaptic plasticity and neuronal proliferation. ${ }^{1}$ Taken together, these data suggest that 48-hour TSD is a significant stressor on central nervous system and systemic function and is consistent with a hypothesis that TSD can compromise neuronal function. Two recent studies of young athletes are of particular relevance in this context because they show that the scores obtained in standard tests used in the evaluation of neurocognitive symptoms of concussion are sensitive to prior sleep history in baseline (non-concussed) subjects. ${ }^{44,45}$ The CSR protocol has also been found to induce long-term alterations in sleep homeostasis in rats, ${ }^{46,47}$ as well as memory and neurobehavioral impairment and effects on immune and peripheral tissue function. ${ }^{48-52}$ Many of the above-mentioned consequences of sleep deprivation are rapidly reversible upon resumption of unrestricted sleep, suggesting that the mechanisms involved in any underlying neuronal dysfunction are reversible. Studies have shown that widespread neuronal degeneration through necrotic and apoptotic mechanisms is not apparent during even prolonged $\mathrm{TSD},{ }^{4,6}$ prompting us to search for signs of an alternative form of neuronal structural impairment as represented by the DN. The data did not support this hypothesis, and we therefore conclude that the mechanisms leading to DN compaction and hyperbasophilia are unaffected by moderate sleep debt in rat cortex.

Furthermore, we show for the first time that the susceptibility of the mechanism leading to DN in response to mild TBI is not strongly affected by acute and chronic sleep debt. We confirmed that the impact model was effective in causing neuronal damage (as reflected by DN density) in the cortex immediately below the point of impact, and we focused our attention on this brain region. A more comprehensive survey of other brain regions may reveal differential effects, but this was not undertaken in the present study. Figure 3 demonstrates that acute and chronic sleep debt did not increase the median DN density following TBI. This was confirmed by the $95 \%$ CIs of the HodgesLehmann estimator, which shows clearly that the small effect size of TSD-TBI and CSR-TBI, relative to TBI alone, cannot be statistically distinguished from zero. We calculate (Statistical Solutions, LLC, Cottage Grove, WI, USA; http://www. statisticalsolutions.net/pss calc.php) using the HCC data (DN density mean \pm standard deviation, $0.1761 \pm 0.1411, \mathrm{n}=12$ ), that the sample size needed to detect a doubling of DN density at statistical power 0.8 is $n=6$. We sampled $n=8,6,13$, and 10 for TSD, TBI, TSD-TBI and CSR-TBI groups, respectively. For these reasons, we conclude that the negative result is genuine and is unlikely to reflect a type II statistical error. In 
this context, it is important to note that prior sleep debt (both TSD and CSR) increased the variance of the response to TBI. This implies that prior sleep debt had a greater effect on some animals than others, the reasons for which are unclear but might relate to different levels of sleep debt achieved. Unfortunately, we did not record sleep in this study (for reasons given earlier), and are unable to evaluate this possibility.

This negative result is consistent with a recent finding that baseline (pre-TBI) sleep is a poor predictor of post-concussion symptoms in collegiate athletes. ${ }^{53}$ Three studies $^{7,35,54}$ in rodents have tested the hypothesis that prior sleep debt influences the severity of neuronal damage resulting from ischemia (stroke), and a fourth study examined the effect of sleep debt on excitotoxicity. ${ }^{55}$ All four studies hypothesized that loss of sleep would worsen the impact of the insult but instead found that sleep debt appeared to protect the tissues through an unknown "preconditioning" mechanism. There were considerable differences between studies in the magnitude of pre-insult sleep debt and the methods used to achieve it, ranging from a brief 6-hour TSD by gentle handling in rats ${ }^{54}$ to 2 days of mainly REMSD using the platform technique in mice, ${ }^{35}$ to 5 days of TSD by the disk-over-water technique in rats, ${ }^{7}$ and 30 days of CSR (4-hour sleep opportunity per day) using a rotating drum. ${ }^{55}$ The consistency of the result is compelling in view of this variation in stimulus. Our data, which examined the effects of non-contusive impact injury, further support the conclusion that preexisting sleep debt does not worsen the immediate consequences of brain injury. However, we did not find evidence that sleep debt suppressed the TBI-mediated formation of DN. An important difference between the present study and those prior studies concerns the experimental timeline. Specifically, we examined the acute effect of TBI by processing the animals immediately after application of the impact, whereas all four of the prior studies allowed 7-8 days of recovery before the animals were killed and brains processed. Those studies therefore examined the combined effects of sleep debt on neuronal susceptibility to insult and potential delayed damage, plus the capacity to recover over the 1 st week. It is possible that postimpact neuronal damage may develop over time in our model of mild TBI, especially if the trauma is associated with undetected cerebrovascular disturbance or cellular energy crisis. ${ }^{29}$ However, the present study was concerned only with the immediate susceptibility of the neurons to impact, which was the principal reason for our choice of the $\mathrm{DN}$ as a marker of cellular damage. It is well known that $\mathrm{DN}$ formation is very rapid, possibly involving a biophysical mechanism of gel-gel phase transition. ${ }^{11,56}$ Indeed, we placed much emphasis on appropriate fixation techniques precisely because the DN formation process is rapid enough to occur within the time needed for complete fixation of brain tissue using PFA fixatives. ${ }^{16-18}$ The positive controls (pin-prick trauma) demonstrated in each case that DN formation was not time limited in our protocol. Thus, we have shown that the immediate neuronal effects of impact are minimally affected by sleep debt, but we cannot exclude the possibility that sleep debt might influence delayed effects. For example, it is possible that sleep debt might confer a protective function in mild TBI by increasing the proportion of cells that recover, a hypothesis that deserves further study.

\section{Conclusion}

Excess sleepiness and sleep deprivation are associated with increased risk of accident, ${ }^{30,31}$ but the present study has shown that sleep deprivation does not worsen the attendant neuronal damage immediately following concussive injury. Furthermore, by taking care to avoid the artifactual formation of DNs, we have confirmed that acute sleep deprivation does not induce neuronal degeneration in otherwise healthy rat cortex. Thus, sleep debt neither causes neuronal cell damage nor exacerbates damage arising from mild traumatic injury.

\section{Acknowledgments}

This study was funded by the Natural Sciences and Research Council of Canada (NSERC) through a Discovery Grant to RS and Postgraduate Scholarship to AMC. NSERC had no involvement in the design, conduct, or interpretation of this study.

We are grateful to Henry Hong for technical assistance and advice, and to Dr John Peever for the use of his microscope and associated equipment.

\section{Disclosure}

Both authors declare no conflict of interest.

\section{References}

1. McCoy JG, Strecker RE. The cognitive cost of sleep lost. Neurobiol Learn Mem. 2011;96(4):564-582.

2. Ribeiro S. Sleep and plasticity. Pflugers Arch. 2012;463(1):111-120.

3. Biswas S, Mishra P, Mallick BN. Increased apoptosis in rat brain after rapid eye movement sleep loss. Neuroscience. 2006;142(2):315-331.

4. Cirelli C, Shaw PJ, Rechtschaffen A, Tononi G. No evidence of brain cell degeneration after long-term sleep deprivation in rats. Brain Res. 1999;840(1-2):184-193.

5. Eiland MM, Ramanathan L, Gulyani S, et al. Increases in amino-cupricsilver staining of the supraoptic nucleus after sleep deprivation. Brain Res. 2002;945(1):1-8.

6. Hipolide DC, D'Almeida V, Raymond R, Tufik S, Nobrega JN. Sleep deprivation does not affect indices of necrosis or apoptosis in rat brain. Int J Neurosci. 2002;112(2):155-166. 
7. Hsu JC, Lee YS, Chang CN, Ling EA, Lan CT. Sleep deprivation prior to transient global cerebral ischemia attenuates glial reaction in the rat hippocampal formation. Brain Res. 2003;984(1-2):170-181.

8. Morrissey MJ, Duntley SP, Anch AM, Nonneman R. Active sleep and its role in the prevention of apoptosis in the developing brain. Med Hypotheses. 2004;62(6):876-879.

9. Rechtschaffen A, Bergmann BM. Sleep deprivation in the rat: an update of the 1989 paper. Sleep. 2002;25(1):18-24.

10. Everson CA, Gilliland MA, Kushida CA, et al. Sleep deprivation in the rat: IX. Recovery. Sleep. 1989;12(1):60-67.

11. Csordas A, Mazlo M, Gallyas F. Recovery versus death of "dark" (compacted) neurons in non-impaired parenchymal environment: light and electron microscopic observations. Acta Neuropathol. 2003;106(1): 37-49.

12. Kherani ZS, Auer RN. Pharmacologic analysis of the mechanism of dark neuron production in cerebral cortex. Acta Neuropathol. 2008;116(4):447-452.

13. Kovesdi E, Pal J, Gallyas F. The fate of "dark" neurons produced by transient focal cerebral ischemia in a non-necrotic and non-excitotoxic environment: neurobiological aspects. Brain Res. 2007;1147 272-283.

14. Ooigawa $H$, Nawashiro H, Fukui S, et al. The fate of Nissl-stained dark neurons following traumatic brain injury in rats: difference between neocortex and hippocampus regarding survival rate. Acta Neuropathol. 2006;112(4):471-481.

15. Crile GW. Studies in exhaustion: an experimental research. Arch Surg. 1921;2(2):196-220.

16. Cammermeyer J. The post-mortem origin and mechanism of neuronal hyperchromatosis and nuclear pyknosis. Exp Neurol. 1960;2: 379-405.

17. Cammermeyer J. The importance of avoiding "dark" neurons in experimental neuropathology. Acta Neuropathol. 1961;1:245-270.

18. Cammermeyer J. Is the solitary dark neuron a manifestation of postmortem trauma to the brain inadequately fixed by perfusion? Histochemistry. 1978;56(2):97-115.

19. Gallyas F, Guldner FH, Zoltay G, Wolff JR. Golgi-like demonstration of "dark" neurons with an argyrophil III method for experimental neuropathology. Acta Neuropathol. 1990;79(6):620-628.

20. Jortner BS. The return of the dark neuron. A histological artifact complicating contemporary neurotoxicologic evaluation. Neurotoxicology. 2006;27(4):628-634.

21. Zsombok A, Toth Z, Gallyas F. Basophilia, acidophilia and argyrophilia of "dark" (compacted) neurons during their formation, recovery or death in an otherwise undamaged environment. J Neurosci Methods. 2005;142(1):145-152.

22. Gallyas F, Zoltay G. An immediate light microscopic response of neuronal somata, dendrites and axons to non-contusing concussive head injury in the rat. Acta Neuropathol. 1992;83(4):386-393.

23. Gallyas F, Zoltay G, Balas I. An immediate light microscopic response of neuronal somata, dendrites and axons to contusing concussive head injury in the rat. Acta Neuropathol. 1992;83(4):394-401.

24. Czurko A, Nishino H. 'Collapsed' (argyrophilic, dark) neurons in rat model of transient focal cerebral ischemia. Neurosci Lett. 1993; 162(1-2):71-74.

25. Golding EM, Robertson CS, Bryan RM Jr. The consequences of traumatic brain injury on cerebral blood flow and autoregulation: a review. Clin Exp Hypertens. 1999;21(4):299-332.

26. Fox CH, Johnson FB, Whiting J, Roller PP. Formaldehyde fixation. Biotech Histochem. 1985;33(8):845-853.

27. Kiernan JA. Fixation of mast cells. Biotech Histochem. 2005;80(1): 44-45.

28. Hyder AA, Wunderlich CA, Puvanachandra P, Gururaj G, Kobusingye OC. The impact of traumatic brain injuries: a global perspective. NeuroRehabilitation. 2007;22(5):341-353.

29. Prins M, Greco T, Alexander D, Giza CC. The pathophysiology of traumatic brain injury at a glance. Dis Model Mech. 2013;6(6): 1307-1315.
30. Strohl KP, Brown DB, Collop N, et al; ATS Ad Hoc Committee on Sleep Apnea, Sleepiness, and Driving Risk in Noncommercial Drivers. An official American Thoracic Society Clinical Practice Guideline: sleep apnea, sleepiness, and driving risk in noncommercial drivers. An update of a 1994 Statement. Am J Respir Crit Care Med. 2013;187(11): 1259-1266.

31. Uehli K, Mehta AJ, Miedinger D, et al. Sleep problems and work injuries: a systematic review and meta-analysis. Sleep Med Rev. 2014;18(1):61-73.

32. Barlow KM. Postconcussion syndrome: a review. J Child Neurol. 2014;29(1):1-11.

33. Gao B, Cam E, Jaeger H, Zunzunegui C, Sarnthein J, Bassetti CL. Sleep disruption aggravates focal cerebral ischemia in the rat. Sleep. 2010;33(7):879-887.

34. Martinez-Vargas M, Estrada Rojo F, Tabla-Ramon E, et al. Sleep deprivation has a neuroprotective role in a traumatic brain injury of the rat. Neurosci Lett. 2012;529(2):118-122.

35. Weil ZM, Norman GJ, Karelina K, et al. Sleep deprivation attenuates inflammatory responses and ischemic cell death. Exp Neurol. 2009;218(1):129-136.

36. Castriotta RJ, Atanasov S, Wilde MC, Masel BE, Lai JM, Kuna ST. Treatment of sleep disorders after traumatic brain injury. J Clin Sleep Med. 2009;5(2):137-144.

37. Caron AM, Stephenson R. Energy expenditure is affected by rate of accumulation of sleep deficit in rats. Sleep. 2010;33(9):1226-1235.

38. Foda MA, Marmarou A. A new model of diffuse brain injury in rats. Part II: morphological characterization. J Neurosurg. 1994;80(2): 301-313.

39. Marmarou A, Foda MA, van den Brink W, Campbell J, Kita H, Demetriadou K. A new model of diffuse brain injury in rats. Part I: pathophysiology and biomechanics. J Neurosurg. 1994;80(2):291-300.

40. Gallyas F. Physicochemical mechanisms of histological silver staining and their utilization for rendering individual silver methods selective and reliable. Biotech Histochem. 2008;83(5):221-238.

41. Gundersen HJ, Bagger P, Bendtsen TF, et al. The new stereological tools: disector, fractionator, nucleator and point sampled intercepts and their use in pathological research and diagnosis. APMIS. 1988;96(10):857-881.

42. Jinno S, Kinukawa N, Kosaka T. Morphometric multivariate analysis of GABAergic neurons containing calretinin and neuronal nitric oxide synthase in the mouse hippocampus. Brain Res. 2001;900(2):195-204.

43. Rechtschaffen A, Bergmann BM, Gilliland MA, Bauer K. Effects of method, duration, and sleep stage on rebounds from sleep deprivation in the rat. Sleep. 1999;22(1):11-31.

44. McClure DJ, Zuckerman SL, Kutscher SJ, Gregory AJ, Solomon GS Baseline neurocognitive testing in sports-related concussions: the importance of a prior night's sleep. Am J Sports Med. 2014;42(2):472-478.

45. Mihalik JP, Lengas E, Register-Mihalik JK, Oyama S, Begalle RL, Guskiewicz KM. The effects of sleep quality and sleep quantity on concussion baseline assessment. Clin J Sport Med. 2013;23(5):343-348.

46. Kim Y, Laposky AD, Bergmann BM, Turek FW. Repeated sleep restriction in rats leads to homeostatic and allostatic responses during recovery sleep. Proc Natl Acad Sci U S A. 2007;104(25):10697-10702.

47. Stephenson R, Caron AM, Famina S. Behavioral sleep-wake homeostasis and EEG delta power are decoupled by chronic sleep restriction in the rat. Sleep. 2015;38(5):685-697.

48. Deurveilher S, Bush JE, Rusak B, Eskes GA, Semba K. Psychomotor vigilance task performance during and following chronic sleep restriction in rats. Sleep. 2015;38(4):515-528.

49. Everson CA, Henchen CJ, Szabo A, Hogg N. Cell injury and repair resulting from sleep loss and sleep recovery in laboratory rats. Sleep. 2014;37(12):1929-1940.

50. McCoy JG, Christie MA, Kim Y, et al. Chronic sleep restriction impairs spatial memory in rats. Neuroreport. 2013;24(2):91-95.

51. Van Dongen HP, Maislin G, Mullington JM, Dinges DF. The cumulative cost of additional wakefulness: dose-response effects on neurobehavioral functions and sleep physiology from chronic sleep restriction and total sleep deprivation. Sleep. 2003;26(2):117-126. 
52. Zager A, Andersen ML, Ruiz FS, Antunes IB, Tufik S. Effects of acute and chronic sleep loss on immune modulation of rats. Am J Physiol Regul Integr Comp Physiol. 2007;293(1):R504-R509.

53. Merritt VC, Arnett PA. Premorbid predictors of postconcussion symptoms in collegiate athletes. J Clin Exp Neuropsychol. 2014;36(10): 1098-1111.

54. Moldovan M, Constantinescu AO, Balseanu A, Oprescu N, Zagrean L, Popa-Wagner A. Sleep deprivation attenuates experimental stroke severity in rats. Exp Neurol. 2010;222(1):135-143.
55. Novati A, Hulshof HJ, Granic I, Meerlo P. Chronic partial sleep deprivation reduces brain sensitivity to glutamate N-methyl-D-aspartate receptor-mediated neurotoxicity. J Sleep Res. 2012;21(1):3-9.

56. Gallyas F, Pal J, Bukovics P. Supravital microwave experiments support that the formation of "dark" neurons is propelled by phase transition in an intracellular gel system. Brain Res. 2009;1270:152-156.

57. Paxinos G, Watson C. The Rat Brain in Stereotaxic Coordinates. 4th ed. San Diego, CA: Academic Press; 1998.

\section{Publish your work in this journal}

Nature and Science of Sleep is an international, peer-reviewed, open access journal covering all aspects of sleep science and sleep medicine, including the neurophysiology and functions of sleep, the genetics of sleep, sleep and society, biological rhythms, dreaming, sleep disorders and therapy, and strategies to optimize healthy sleep. The journal welcomes

\section{Dovepress}

original research, clinical \& epidemiological studies, reviews \& evaluations, case reports and extended reports. The manuscript management system is completely online and includes a very quick and fair peerreview system, which is all easy to use. Visit http://www.dovepress.com/ testimonials.php to read real quotes from published authors. 